\title{
Putting Yourself in the Skin of a Black Avatar Reduces Implicit Racial Bias
}

\author{
Tabitha C. Peck ${ }^{1+}$, Sofia Seinfeld ${ }^{1,2}$, Salvatore M Aglioti ${ }^{3}$, Mel Slater ${ }^{1,4,5^{*}}$ \\ ${ }^{1}$ Universitat de Barcelona \\ Event Lab, Facultat de Psicologia \\ Campus de Mundet - Edifici Teatre \\ Passeig de la Vall d'Hebron 171 \\ 08035 Barcelona \\ Spain \\ Email: tpeck@cs.duke.edu \\ ${ }^{2}$ Institut de Investigacions Biomèdiques August Pi i Sunyer (IDIBAPS) \\ Rosselló, 149-153 \\ 08036, Barcelona \\ Spain \\ Email: seinfeld@clinic.ub.es \\ ${ }^{3}$ Department of Psychology, Sapienza University of Rome and Fondazione Santa Lucia \\ IRCCS \\ I-00179 Roma \\ Italy \\ Email: salvatoremaria.aglioti@uniroma1.it \\ ${ }^{4}$ Institució Catalana de Recerca i Estudis Avançats (ICREA) \\ Passeig Lluís Companys, 23 \\ 08010 Barcelona \\ Spain \\ ${ }^{5}$ Department of Computer Science \\ University College London \\ Gower Street \\ London WC1E 6BT \\ UK
}

*Corresponding Author:

Email: melslater@ub.edu

Tel: +34934039618

\footnotetext{
${ }^{+}$Currently at Department of Computer Science, Duke University, LSRC Building D101 308 Research Drive, Campus Box 90129, Durham, NC 27708, USA.
} 


\begin{abstract}
Although it has been shown that immersive virtual reality (IVR) can be used to induce illusions of ownership over a virtual body (VB), information on whether this changes implicit interpersonal attitudes is meager. Here we demonstrate that embodiment of light-skinned participants in a dark-skinned VB significantly reduced implicit racial bias against darkskinned people, in contrast to embodiment in light-skinned, purple-skinned or with no VB. 60 females participated in this between-groups experiment, with a VB substituting their own, with full-body visuomotor synchrony, reflected also in a virtual mirror. A racial Implicit Association Test (IAT) was administered at least three days prior to the experiment, and immediately after the IVR exposure. The change from pre- to post-experience IAT scores suggests that the dark-skinned embodied condition decreased implicit racial bias more than the other conditions. Thus, embodiment may change negative interpersonal attitudes and thus represent a powerful tool for exploring such fundamental psychological and societal phenomena.
\end{abstract}

\title{
Keywords
}

Racial bias, Implicit Association Test, IAT, virtual reality, virtual environment, body ownership, embodiment

\section{Introduction}

There is mounting evidence that virtual reality techniques can be used to produce a strong ownership illusion over a virtual body (Ehrsson, 2007; Lenggenhager, Tadi, Metzinger, \& Blanke, 2007; Petkova \& Ehrsson, 2008) even when there are radical changes in comparison to the true body (Kilteni, Normand, Sanchez Vives, \& Slater 2012; Normand, Giannopoulos, Spanlang, \& Slater, 2011; van der Hoort, Guterstam, \& Ehrsson, 2011). However, information as to whether transformation in body representation can change interpersonal attitudes is meager. There is some evidence that virtual alteration of age through embodiment in an elderly person can reduce negative stereotypes toward the elderly (Yee \& Bailenson, 2007) and also that embodying men in a female child virtual body produces a strong physiological response when the child is placed in a threatening situation (Slater, Spanlang, Sanchez-Vives, \& Blanke, 2010). 
Our specific concern here is the hostility toward 'out-groups' that seems to be present in all human cultures. Hostility towards racial out-groups in particular seems so ingrained in human nature that it is at play even when interacting with virtual characters. It has been shown, for example, that people are less likely to help black avatars (Eastwick \& Gardner, 2009) and show more aggression toward them than white avatars (McCall, Blascovich, Young, \& Persky, 2009).

Encoding people by race may be a reversible by product of human evolution used to detect coalitional alliances (Kurzban, Tooby, \& Cosmides, 2001). This carries the remarkable implication that racism might be overcome in a number of circumstances. For example, evidence has suggested that the sensation of ownership over a black rubber arm by White subjects may be associated with a change in implicit racial bias (Farmer, Tajadura-Jimenez, \& Tsakiris, 2012; Maister, Sebanz, Knoblich, \& Tsakiris, 2013). Further, it has been shown that constructing artificially a mixed-race coalitional alliance with still photographs that depicted members of two teams, led to enhanced positive automatic evaluation of Black in-group (same team) members by White in-group members in the absence of any effect on White participant views of Black out-group (other team) members (Kurzban, et al., 2001; Van Bavel \& Cunningham, 2009).

Immersive Virtual Reality (IVR) provides a powerful tool for potentially placing people into a different 'coalition', here specifically race, by changing the form of their body representation. This is achieved by a setup that we refer to as 'virtual embodiment'. The participants wear a wide field-of-view head tracked head mounted display. When they look down towards themselves in the VR they would see a programmed virtual body (VB) substituting their own real body. They would also see this body when looking at their (geometrically correct) reflection in a virtual mirror. Additionally, participants wear a bodytracking suit that provides real-time motion capture. So as they move their real body they would see their VB move synchronously. By embodying participants in bodies of different skin color we sought to determine whether (i) we could induce a body-ownership illusion in a differently raced avatar, and (ii) whether the body-ownership illusion could reduce negative implicit responses toward that other race.

\section{Materials and Methods}

\subsection{Experimental Design}

The experiment reported in this paper was a between-groups design including 60 lightskinned female participants of Spanish origin recruited from the same population of female students at the University of Barcelona, none of whom had any connection with our laboratories. They were distributed arbitrarily into 4 conditions of 15 each: (EL) embodied- 
light or (ED) embodied-dark or (ND) non-embodied-dark or (EA) embodied-alien. Specifically (see Fig. 1),

- Embodied-Light-Skinned (EL) - Here participants were embodied in a light-skinned virtual body (VB). Embodiment was achieved through visual-motor synchrony between the real and VB that could be seen either directly when looking towards the own body, and in a reflection in a virtual mirror.

- Embodied-Dark-Skinned (ED) - This was identical to the previous one except that the VB was dark-skinned.

- Non-Embodied Dark-Skinned (ND) - In this case there was no VB substituting for the participant's own body, and the VB seen in the mirror, although in the geometrically correct place and of size to be a mirror reflection, moved asynchronously with respect to the participant's movements. The body seen in the mirror was dark-skinned.

- Embodied-Alien-Skinned (EA). Here the virtual body was colored in a bright medium toned purple color that was alien and unnatural for people. We also refer to the skin color as purple.

In each condition participants experienced the same events always from the same visual perspective. We included the last condition it order to check whether any effects we might find were specifically due to the effect of the dark-skinned color (i.e., different human race), or only because it was different (not specifically related to race).

\subsection{Procedures}

On a first visit to the laboratory participants completed an Implicit Association Test (IAT) (Greenwald, McGhee, \& Schwartz, 1998) on a laptop and the results were recorded (variable preIAT). After a period of at least three days they returned for the main experiment. They put on a wide field-of-view, stereo head-tracked head-mounted display through which they would find themselves in the center of a $14 \mathrm{~m}$ by $3 \mathrm{~m}$ long hallway with a mirror on one wall. For those in the embodied conditions (EL, ED, EA) the scene included a virtual body that substituted their own viewed from first person perspective (1PP). Additionally, they wore a motion capture suit so that when they moved their real body, the VB moved correspondingly in real-time. They saw their VB when looking directly down at themselves toward their real (but unseen) body, and also when looking in the virtual mirror. Those in the non-embodied (ND) condition wore the same equipment, and saw the same environment and events, from the same viewpoint and perspective, including a dark-skinned body in the virtual mirror at the correct place to represent a mirror reflection. For these participants there was no VB that 
substituted their own body, and the mirror body moved independently of their own movements.

There was a 5-minute embodiment phase where participants in all experimental conditions were asked to look around the environment, look in the mirror, explore and move their virtual body, and describe their surroundings and body. This was followed by a 6.5 minute approach phase when 12 virtual human female characters (6 light and 6 dark-skinned) walked past them one by one, either to the right or left.

Finally the IAT was administered in the HMD using all female faces of the same type as the avatars created for the virtual environment, and the same as those used in the initial administration of the IAT. We call the results of this the postIAT variable. Participants then took off the HMD and completed a questionnaire about their experience (Table 1) including questions about the level of subjective body ownership (mybody), how nervous they were when the virtual characters approached them (nervous), and an explicit racial bias questionnaire. The scenario and equipment is illustrated in Fig. 1 and Supplementary Video S1.

\subsection{Equipment}

For the virtual reality session participants were fitted with a stereo NVIS nVisor SX111 headmounted display (HMD). This has dual SXGA displays with $76^{\circ} \mathrm{H} \times 64^{\circ} \mathrm{V}$ degrees field of view (FOV) per eye, totaling a wide field-of-view of $111^{\circ}$ horizontal and $60^{\circ}$ vertical, with a resolution of $1280 \times 1024$ per eye displayed at $60 \mathrm{~Hz}$. Head tracking was performed by a 6 DOF Intersense IS-900 device.

The participants wore a tight fitting Velcro suit that had retro-reflective markers attached that enabled whole body tracking. The marker-based infrared tracking system was a 12 camera Optitrack system from Naturalpoint ${ }^{1}$ that could track a volume of approximately $2.5 \mathrm{~m}$ width $\times 2.5 \mathrm{~m}$ length $\times 3 \mathrm{~m}$ height. $2 \mathrm{D}$ marker information was transferred from the cameras via USB to the NaturalPoint Arena motion capture software in which participant's dynamic skeletal configurations were reconstructed. The movements are reconstructed at $100 \mathrm{~Hz}$ with millimeter accuracy. From Arena the skeletal motion data was streamed to a hardware accelerated character animation system via the NatNet protocol. The skeletal motion data was then mapped so that the Avatar posture matched that of our participant to a good degree. The software used was XVR for the overall virtual reality system (Tecchia, et al., 2010), and HALCA for the character animation (Gillies \& Spanlang, 2010).

\footnotetext{
${ }^{1} \mathrm{http} / / /$ www.naturalpoint.com/optitrack
} 


\subsection{Response Variables}

\subsubsection{Racial Implicit Association Test}

Participants' implicit racial bias was calculated by the administration of a racial IAT (Greenwald, McGhee et al. 1998) twice, one at the first visit to the laboratory and the second immediately after completion of the virtual reality scenario, but while still wearing the HMD.

A racial IAT measures bias by requiring people to quickly categorize faces (black or white) and words (positive or negative) into groups. Implicit bias is calculated from the differences in speed and accuracy between categorizing (white faces, positive words) and (black faces, negative words) compared to (black faces, positive words) and (white faces, negative words). All IAT scores were calculated using the method described in (Greenwald, Nosek, \& Banaji, 2003) which results in a smaller correlation between repeated test scores compared to previous IAT calculations. Repeat IAT scores are known to decrease (Greenwald \& Nosek, 2001); therefore we expected to see a small reduction on the average in the second measurement. The IAT scores are dimensionless, but they are a summary measure of the differential reaction times and accuracy of detection for the categorizations above. Higher IAT is interpreted as greater racial bias, that is, longer reaction times and greater inaccuracies in categorizing black faces with positive words and white faces with negative words than black faces with negative and white faces with positive words. It should be noted that the procedures for producing the final scores were carried out independently by two different investigators for cross validation.

Our interest focused primarily on the difference between the post- and pre-IAT scores, i.e., $\triangle I A T=$ postIAT - preIAT . Our hypothesis was that mean $\triangle I A T$ would show a greater decrease for the participants in ED condition than in the other conditions, where a greater IAT score means greater racial bias against dark-skinned people.

\subsubsection{Post Experience Questionnaire}

After participants had removed the HMD they were asked to complete the questionnaire shown in Table 1. This included 6 statements answered on a 1-5 scale, where 1 meant least and 5 greatest agreement. The four most important questions were Q1 (mybody) assessing the extent of body ownership, Q3 (control) referring to the sensation of agency or control over the virtual body, and Q4 (notme) also assessing body ownership. Q2 (nervous) was a simple way to assess the positive or negative affect associated with the experience of the avatars walking by. The remaining questions were 'filler' questions.

Participants also completed an explicit racial bias questionnaire based on the questionnaire used in (Avenanti, Sirigu, \& Aglioti, 2010) adapted for Spain and shown in 
Table S1. Each question was scored with a Yes or No answer, and the result of the questionnaire was the sum of these responses.

\section{Results}

\subsection{Questionnaire Results}

The question on control (Q3) can be considered as a consistency check, since it was in fact the case that in the embodied conditions the participant could control the body and in the non-embodied condition they could not. The proportions that scored at least 4 out of the maximum of 5 on control were 13/15 (EL), 12/15 (ED), 3/15 (ND) and 15/15 (EA).

The embodiment procedure turned out to be effective with respect to the illusion of body ownership. The scores on ownership with respect to the avatar were significantly lower in the non-embodied (ND) compared to the three embodied conditions (ED, EW and EA), which in turn did not differ from one another.

The two questions that relate to body ownership in Table 1 are Q1 (mybody) affirming body ownership and Q4 (notme) denying it. We can combine the scores on these two variables by taking $(\mathrm{Q} 1+6-\mathrm{Q} 4) / 2$ to obtain an overall score. Fig. 2 shows the means and standard errors of the resulting variable. Analysis of variance between the four conditions shows a significant difference between the means $\left(F(3,56)=4.86, P=0.0045, R^{2}=0.21\right)$. The residual errors of the fit are compatible with normality (Shapiro-Wilk $\mathrm{P}=0.27$ ). Multiple pair-wise comparisons (Duncan's method) with an overall 5\% level of significance shows that ND is significantly less than each of the others, but that there is no significant difference between any of ED, EL, EA as suggested by Fig. 2. An alternative more detailed analysis of the questionnaire responses is given in Supplementary Text S2.

\subsection{Racial Bias}

As expected on the basis of previous literature (Avenanti, et al., 2010), an explicit measure of racial bias resulted in very low scores. Of the 7 questions (with yes/no answers, yes indicating more bias) shown in Supplementary Table S1, the median number of 'yes' answers was 0 with interquartile range (IQR) 1.

The mean preIAT scores were similar across the four conditions (Supplementary Text S2.2, Fig. S2). The means and standard errors of $\triangle I A T$ are shown in Fig. 3. There is a striking difference between the mean ED and the others, in line with our hypothesis. However, it can also be seen that the variance for EA is very large and the variance ratio of ED to EA is significantly different from 1 at $\mathrm{P}=0.03$ ( $\mathrm{F}=0.30$ on $(14,14)$ d.f., two-sided, with both variables compatible with normality, Shapiro-Wilk $\mathrm{P}=0.10$ for ED and 0.66 for EA). The high variance of EA indicates that a prediction of $\triangle I A T$ for someone in this condition has far 
greater uncertainty attached to it than a prediction for the ED condition, where it would be a safe bet that $\triangle I A T<0$.

The questionnaire response nervous unexpectedly played an important role in the results since the greater the degree of nervousness the more likely that postIAT would be less than preIAT. Fig. 4 shows this in the scatter plots of $\triangle I A T$ against nervous, suggesting a negative association in all the conditions except EL.

Now we compare $\triangle I A T$ between EL and ED. One way ANOVA indicates that the means are significantly different $\left(F(1,28)=5.01, P=0.033, \eta^{2}=R^{2}=0.15\right.$, SW test for normality of residuals $\mathrm{P}=0.06$ ). Including also nervous as a covariate there is a significant interaction term, which can also be seen in Fig. 4a,b $\left(\mathrm{F}(1,26)=4.38, \mathrm{P}=0.046\right.$, partial $\eta^{2}=0.15, \mathrm{R}^{2}=$ $0.33, \mathrm{SW} P=0.49$ ). Estimating the marginal (over nervous) means then gives for EL mean \pm S.E.: $0.049 \pm 0.064(\mathrm{P}=0.44)$ and for ED $-0.187 \pm 0.061(\mathrm{P}=0.002)$ and the confidence intervals for these two means slightly overlap (by 0.03 ) at the $95 \%$ level but not at the $93 \%$ level.

The difference between the means of ED and ND is not significant (ANOVA $F(1,28)=$ $1.46, \mathrm{P}=0.24$ ) but when the covariate nervous is introduced then there is a dramatic change. $\mathrm{R}^{2}=0.37$, the coefficient of nervous is negative $\left(\mathrm{F}(1,27)=13.74, \mathrm{P}=0.001\right.$, partial $\left.\eta^{2}=0.34\right)$ and $\mathrm{ED}$ has a significantly lower intercept than $\mathrm{ND}(\mathrm{F}(1,27)=5.79, \mathrm{P}=0.023)(\mathrm{SW} \mathrm{P}=$ 0.44, partial $\eta^{2}=0.18$ ). Again looking at the marginal mean estimates, for ED: $-0.168 \pm 0.057(P=0.003)$, and for ND: $0.028 \pm 0.057(P=0.617)$, but the two $95 \%$ confidence intervals for the means do slightly overlap (by a value of 0.03 ), but not at the $91 \%$ level.

Overall we find that those in the ED group tend to have a lower level of IAT after the exposure. Moreover, for ED, ND and EA groups the questionnaire response nervous is negatively associated with $\triangle I A T$. Provided that the covariate nervous is taken into account, the hypothesis is supported: $\triangle I A T$ for ED is less than for the ND condition and the EL condition, and in comparison to the EA condition it has a significantly smaller variance (making comparison of the means not useful).

\section{Discussion}

A corollary to the issue of body ownership illusions is the extent to which virtual embodiment can change implicit biases such as racial prejudice, that are likely rooted in our personality and that can be modified only with great effort. Here we have demonstrated a whole body ownership illusion where IVR was used to enable people to step into the 'skin' of 
a differently raced body. More importantly, we demonstrate that embodiment of light-skinned individuals in a dark-skinned virtual body at least temporarily reduces their implicit bias against people who are coded as out-group on the basis of the color of the skin. This effect appears to be specifically linked to racial bias since embodiment in an alien, purple skin virtual body towards which no stereotypes or prejudices can be automatically associated, did not result in the same response.

However, the results should be treated with caution, since the difference in the level of body ownership between the non-embodied (ND) and the embodied conditions (E*) was significant but not great. It is possible that simply seeing the dark skinned avatar in the mirror, located at the correct place for a reflection, might have been enough to generate an illusion of body ownership. Moreover, although the most important comparison of $\triangle I A T$, between ED and EL, was significant, other differences were mediated through the variable nervous.

Generally the variable nervous has figured prominently in our results. Indeed it could be the case that it is not embodiment in a dark-skinned VB per se that is responsible for the results but nervousness due to such embodiment. There is evidence to suggest that anxiety enhances aversive processing (Robinson, Charney, Overstreet, Vytal, \& Grillon, 2012; Robinson, Letkiewicz, Overstreet, Ernst, \& Grillon, 2011) so that one explanation for our results could be as follows: when people with racial bias are embodied in a dark-skinned body this makes them nervous, which then speeds up their responses, which therefore lowers the IAT score. However, this explanation appears not to hold. The partial correlation between $\triangle I A T$ and condition $(\mathrm{EL}=0, \mathrm{ED}=1)$ eliminating nervous is $\mathrm{r}=-0.47(\mathrm{P}=0.01)$. Similarly, the partial correlation between $\triangle I A T$ and condition $(\mathrm{ED}=1, \mathrm{ND}=2)$ is $\mathrm{r}=0.42(\mathrm{P}=0.02)$.

The variable nervous ("I became nervous when the other avatars approached me") might simply reflect the impact of the scenario on the participants with nervousness acting as a surrogate for presence, the sensation of being in the virtual scenario (Sanchez-Vives \& Slater, 2005). This could be the case since the virtual characters that walked by the participant clearly entered their proxemics intimate zone (Hall, 1969), and proxemics is known to operate in IVR (Bailenson, Blascovich, Beall, \& Loomis, 2003; Llobera, Spanlang, Ruffini, \& Slater, 2010). A hypothesis would be that the greater the presence, the more effective the experimental condition of embodiment in the dark-skinned VB, therefore leading to a reduction in racial bias scores. Hence it is possible that the underlying causal variable may be presence rather than nervousness itself, but we are not able to test this.

Our results expand previous work on the use of explicit strategies such as perspective taking or role-playing (Todd, Bodenhausen, \& Galinsky, 2012; Todd, Bodenhausen, Richeson, \& Galinsky, 2011), voluntary mimicry (Inzlicht, Gutsell, \& Legault, 2012) or explicit non-verbal behavior (Castelli, Carraro, Pavan, Murelli, \& Carraro, 2012), for 
reducing indices of racial bias by demonstrating that virtual embodiment can induce changes in implicit attitudes in the absence of explicit priming for perspective taking or role-playing.

Our result stands in contrast with the only previous attempt to explore whether virtual reality may change implicit attitudes in which embodiment in a Black virtual body apparently increased racial bias (Groom, Bailenson, \& Nass, 2009). However, the issue of body ownership was not considered, there was no full body visuomotor synchrony but only correlated head movements, and the embodiment phase was very short lasting only 60-75 seconds (while it is 11.5 minutes in our study). Critically, participants were told to imagine and role-play a day in the life of the individual and were placed into a situation where the embodied individual was applying for a job, a situation that is known for race discrimination (Dasgupta, 2004). Pre-exposure to information can influence IAT scores (Blair, 2002) and we hypothesize that the increase in implicit racial bias scores found in the study of Groom et al. (2009) was due not to embodiment, but to the pre-exposure of being placed into a situation that is known for race discrimination. In our study the participants were given no task at all, except for the 5 minutes of observing their environment and virtual body, both directly by looking towards their body and in a mirror, and then watching the 12 virtual characters walk by.

Our results can also be compared with studies that examined the extent to which White participants could experience ownership over a black rubber hand (and a hand presented in video) (Farmer, et al., 2012; Maister, et al., 2013). It was found that ownership over a black rubber hand was induced through synchronous visual-tactile stimulation over the seen rubber hand and the corresponding obscured real hand, as in the normal case of the rubber hand illusion (Botvinick \& Cohen, 1998) independently of the color of the hand (black or white). However, it was also noted that based on subjective questionnaire responses the reported strength of such ownership was lower in the black compared to the white rubber hand condition, and the level of ownership of the black hand seen in video was generally very low. It was also found that the level of ownership predicted a lower post-experiment racial IAT score compared to the pre-experiment score, but that there was no difference due to the actual experimental condition. The results of the current study are consistent with this earlier work. However, there are some interesting discrepancies. First, in our study there was no difference in the level of whole body ownership between the embodied conditions (EL, ED, EA) irrespective of skin color. This is probably due to the powerful effect of the first person perspective experience of the virtual body (Petkova, Khoshnevis, \& Ehrsson, 2011; Slater, et al., 2010) seen both directly and in the mirror, and the synchronous visuomotor correlation between the real and virtual body movements (Gonzalez-Franco, Perez-Marcos, Spanlang, \& Slater 2010; Kalckert \& Ehrsson, 2012; Sanchez-Vives, Spanlang, Frisoli, Bergamasco, \& 
Slater 2010). Second, the direct manipulation in our experiment did produce a change in IAT: actual embodiment (1PP) in a dark-skinned body was more likely to produce an IAT change than non-embodiment or embodiment in a light-skinned body (taking into account the covariate nervous). Third, our 'alien' (purple-skinned) condition allowed for the possibility that it was simply difference or strangeness that could account for the results rather than the specific issue of race.

How is it possible that a few minutes exposure to a dark-skinned virtual body seen from first person perspective can have an effect on something seemingly completely unrelated essentially reaction times in classifying images and words on the basis of race? In (Kurzban, et al., 2001; Van Bavel \& Cunningham, 2009) it was shown that the formation of new ingroup and out-groups based simply on pictures could lessen racial bias towards black members of the in-group. Our method provides a quite unique and novel way to temporarily transfer someone to a different in-group - and based directly on a critical aspect of the appearance of members of that group (skin color). This could be argued to be a very powerful way of transforming group affiliation. In (Richeson \& Shelton, 2003) it was shown that interaction of White students with a Black confederate experimenter led to worse subsequent performance on a Stroop test compared to those who interacted with a White confederate, and that performance was correlated with a pre-experiment IAT test (greater racial bias predicting worse Stroop test performance). It was argued that the results were consistent with a resource model of executive control, where the deployment of resources involved in coping with the interracial interaction was detrimental to the performance of the Stroop task, a result that received backing in a later fMRI study (Richeson, et al., 2003). This is also consistent with the results of our study. For those in the ED condition, with their affiliation temporarily transferred to the racial out-group there could be less resource expended to cope with the events of the nominally out-group virtual humans walking past them, since in the ED condition these may be not be perceived as out-group.

\section{Conclusions}

The results from our experiment, and also earlier work discussed above, shows that IVR can be used to generate an illusion of body ownership through first person perspective of a virtual body that substitutes their own body. Provided that there is no body movement this is enough to generate this illusion (Maselli \& Slater, 2013). Other multisensory feedback, such as visuomotor synchrony as used in our experiment, may heighten this illusion. The main focus of interest here is that virtual bodies may have a particular 'semantics' associated with them - possibly through stereotyping. This 'body semantics' may have behavioural correlates for the participant - for example, it has been shown that being embodied in a dark-skinned 
casual looking virtual body leads to more expressive drumming performance than being embodied in a light-skinned formally dressed one (Kilteni, Bergstrom, \& Slater, 2013). In this paper we have pointed to a further consequence of body semantics beyond a behavioural effect to a far more complex one involving apparently deep seated issues such as racial bias, with results suggesting that embodiment of light-skinned people in a dark skinned body can lead to a comparative reduction in their implicit racial bias. The next question to consider is how long this effect lasts after the experimental manipulation - whether it is short- or longlasting.

\section{Acknowledgements}

This research was supported by VERE, an Integrated Project funded under the European Seventh Framework Program, Future and Emerging Technologies (FET), Grant Agreement Number 257695, and the European Research Council project TRAVERSE (Grant Agreement Number 227985). We would like to thank Xavier Navarro and Ausias Pomes for helping with images and videos.

\section{References}

Avenanti, A., Sirigu, A., \& Aglioti, S. M. (2010). Racial bias reduces empathic sensorimotor resonance with other-race pain. Current Biology, 20(11), 1018-1022.

Bailenson, J., Blascovich, J., Beall, A., \& Loomis, J. (2003). Interpersonal Distance in Immersive Virtual Environments. Personality and Social Psychology Bulletin, 29(7), 819-833.

Blair, I. V. (2002). The malleability of automatic stereotypes and prejudice. Personality and Social Psychology Review, 6(3), 242-261.

Botvinick, M., \& Cohen, J. (1998). Rubber hands 'feel' touch that eyes see. Nature, 391(6669), 756-756.

Castelli, L., Carraro, L., Pavan, G., Murelli, E., \& Carraro, A. (2012). The Power of the Unsaid: The Influence of Nonverbal Cues on Implicit Attitudes. Journal of Applied Social Psychology(DOI: 10.1111/j.1559-1816.2012.00903.x).

Dasgupta, N. (2004). Implicit ingroup favoritism, outgroup favoritism, and their behavioral manifestations. Social Justice Research, 17(2), 143-169.

Eastwick, P. W., \& Gardner, W. L. (2009). Is it a game? Evidence for social influence in the virtual world. Social Influence, 4(1), 18-32.

Ehrsson, H. H. (2007). The experimental induction of out-of-body experiences. Science, 317(5841), 1048-1048.

Farmer, H., Tajadura-Jimenez, A., \& Tsakiris, M. (2012). Beyond the colour of my skin: how skin colour affects the sense of body-ownership. Consciousness and Cognition, 21(3), 1242-1256.

Gillies, M., \& Spanlang, B. (2010). Comparing and evaluating real-time character engines for virtual environments. PRESENCE: Teleoperators and Virtual Environments, 19(2), 95-117.

Gonzalez-Franco, M., Perez-Marcos, D., Spanlang, B., \& Slater , M. (2010). The Contribution of Real-Time Mirror Reflections of Motor Actions on Virtual Body 
Ownership in an Immersive Virtual Environment Paper presented at the IEEE Virtual Reality.

Greenwald, A. G., McGhee, D. E., \& Schwartz, J. L. K. (1998). Measuring individual differences in implicit cognition: The implicit association test. Journal of Personality and Social Psychology, 74(6), 1464.

Greenwald, A. G., \& Nosek, B. A. (2001). Health of the Implicit Association Test at age 3. Zeitschrift $f{ }^{\prime}{ }^{\circ}$ Experimentelle Psychologie, 48(2), 85-93.

Greenwald, A. G., Nosek, B. A., \& Banaji, M. R. (2003). Understanding and using the Implicit Association Test: I. An improved scoring algorithm. Journal of Personality and Social Psychology, 85(2), 197.

Groom, V., Bailenson, J. N., \& Nass, C. (2009). The influence of racial embodiment on racial bias in immersive virtual environments. Social Influence, 4(3), 231-248.

Hall, E. T. (1969). The hidden dimension: Anchor Books New York.

Inzlicht, M., Gutsell, J. N., \& Legault, L. (2012). Mimicry reduces racial prejudice. Journal of Experimental Social Psychology, 48(1), 361-365.

Kalckert, A., \& Ehrsson, H. H. (2012). Moving a rubber hand that feels like your own: a dissociation of ownership and agency. Frontiers in Human Neuroscience, 6(40), doi: 10.3389/fnhum.2012.00040.

Kilteni, K., Bergstrom, I., \& Slater, M. (2013). Drumming in immersive virtual reality: the body shapes the way we play. Transactions on Visualization and Computer Graphics (IEEE), 19(4), 597-605.

Kilteni, K., Normand, J.-M., Sanchez Vives, M. V., \& Slater , M. (2012). Extending Body Space in Immersive Virtual Reality: A Very Long Arm Illusion. PLoS ONE, 7(7), e40867.

Kurzban, R., Tooby, J., \& Cosmides, L. (2001). Can race be erased? Coalitional computation and social categorization. PNAS 98(26), 15387-15392.

Lenggenhager, B., Tadi, T., Metzinger, T., \& Blanke, O. (2007). Video ergo sum: Manipulating bodily self-consciousness. Science, 317(5841), 1096-1099.

Llobera, J., Spanlang, B., Ruffini, G., \& Slater, M. (2010). Proxemics with Multiple Dynamic Characters in an Immersive Virtual Environment ACM Transactions on Applied Perception (TAP), 8(1), Article 3.

Maister, L., Sebanz, N., Knoblich, G., \& Tsakiris, M. (2013). Experiencing ownership over a dark-skinned body reduces implicit racial bias. Cognition, in press.

Maselli, A., \& Slater, M. (2013). The building blocks of the full body ownership illusion. Frontiers in Human Neuroscience, 7(83).

McCall, C., Blascovich, J., Young, A., \& Persky, S. (2009). Proxemic behaviors as predictors of aggression towards Black (but not White) males in an immersive virtual environment. Social Influence, 4(2), 138-154.

Normand, J. M., Giannopoulos, E., Spanlang, B., \& Slater, M. (2011). Multisensory Stimulation Can Induce an Illusion of Larger Belly Size in Immersive Virtual Reality. PLOS ONE, 6(1), e16128. doi:16110.11371/journal.pone.0016128.

Petkova, V. I., \& Ehrsson, H. H. (2008). If I Were You: Perceptual Illusion of Body Swapping. PLoS ONE, 3(12): e3832. doi:10.1371/journal.pone.0003832.

Petkova, V. I., Khoshnevis, M., \& Ehrsson, H. H. (2011). The perspective matters! Multisensory integration in ego-centric reference frames determines full-body ownership. Frontiers in Psychology, 2, Article 35.

Richeson, J. A., Baird, A. A., Gordon, H. L., Heatherton, T. F., Wyland, C. L., Trawalter, S., et al. (2003). An fMRI investigation of the impact of interracial contact on executive function. Nature Neuroscience, 6(12), 1323-1328.

Richeson, J. A., \& Shelton, J. N. (2003). When Prejudice Does Not Pay Effects of Interracial Contact on Executive Function. Psychological Science, 14(3), 287-290.

Robinson, O. J., Charney, D. R., Overstreet, C., Vytal, K., \& Grillon, C. (2012). The adaptive threat bias in anxiety: Amygdala, Äìdorsomedial prefrontal cortex coupling and aversive amplification. Neuroimage, 60(1), 523-529. 
Robinson, O. J., Letkiewicz, A. M., Overstreet, C., Ernst, M., \& Grillon, C. (2011). The effect of induced anxiety on cognition: threat of shock enhances aversive processing in healthy individuals. Cognitive, Affective, \& Behavioral Neuroscience, 11(2), 217-227.

Sanchez-Vives, M. V., \& Slater, M. (2005). From Presence to Consciousness through Virtual Reality. Nature Reviews Neuroscience, 6(4), 332-339.

Sanchez-Vives, M. V., Spanlang, B., Frisoli, A., Bergamasco, M., \& Slater , M. (2010). Virtual hand illusion induced by visuomotor correlations. PLOS ONE, 5(4), e10381, doi:10310.11371/journal.pone.0010381.

Slater, M., Spanlang, B., Sanchez-Vives, M., \& Blanke, O. (2010). First person experience of body transfer in virtual reality. PLoS ONE, 5(5), e10564. doi:10510.11371/journal.pone.0010564.

Tecchia, F., Carrozzino, M., Bacinelli, S., Rossi, F., Vercelli, D., Marino, G., et al. (2010). A flexible framework for wide-spectrum vr development. Presence: Teleoperators and Virtual Environments, 19(4), 302-312.

Todd, A. R., Bodenhausen, G. V., \& Galinsky, A. D. (2012). Perspective-taking combats the denial of intergroup discrimination. Journal of Experimental Social Psychology, 48(3), 738-745.

Todd, A. R., Bodenhausen, G. V., Richeson, J. A., \& Galinsky, A. D. (2011). Perspective taking combats automatic expressions of racial bias. Journal of Personality and Social Psychology, 100(6), 1027-1042.

Van Bavel, J. J., \& Cunningham, W. A. (2009). Self-categorization with a novel mixed-race group moderates automatic social and racial biases. Personality and Social Psychology Bulletin, 35(3), 321-335.

van der Hoort, B., Guterstam, A., \& Ehrsson, H. H. (2011). Being Barbie: the size of one's own body determines the perceived size of the world. PLOS ONE, 6(5), e20195. doi:20110.21371/journal.pone.0020195.

Yee, N., \& Bailenson, J. N. (2007). The Proteus effect: Self transformations in virtual reality. Human Communication Research, 33(3), 271-290. 


\section{Figures}

Fig. 1 - The virtual body and scenario. (a) The light-skinned virtual body (EL) as seen in the mirror. (b) The dark-skinned virtual body (ED) in the mirror and directly. (c) The purpleskinned body (EA) as seen in the mirror. (d) A dark-skinned virtual character walks by - the first person viewpoint and corresponding view in the mirror for the ED condition. (e) A participant wearing the HMD and body tracking suit.

Fig. 2 - Bart chart showing means and standard errors of average embodiment questionnaire score by condition. The average embodiment score is obtained as $(\mathrm{Q} 1+6-\mathrm{Q} 4) / 2$. Q1 (mybody) affirms body ownership and Q4 (notme) denies it through affirming that the virtual body was 'not me' (Table 1).

Fig. 3 - Bar chart showing means and standard errors of $\triangle I A T$ by condition.

Fig. 4 - Scatter diagram of $\triangle I A T$ by nervous for each condition. For EL the slope is positive but not significantly different from $0\left(\mathrm{R}^{2}=0.03, \mathrm{P}=0.57\right)$. For the remainder the slopes are negative with significance levels $E D R^{2}=0.39, P=0.014$; ND $R^{2}=0.30, P=0.035$; and for $E A R^{2}=0.19, P=0.109$. In each case the residual errors are compatible with normality (for EL Shapiro-Wilk $\mathrm{P}=0.16$, and for the others all $\mathrm{P}>0.94$ ). ANCOVA of $\triangle I A T$ on condition with nervous as the covariate shows a negative slope for nervous $(\mathrm{P}=0.002)$ and with $\mathrm{ED}$ having a significantly lower intercept than EL $(P=0.009)$ but no other significant differences, with $R^{2}=0.20 . P=0.30$ for normality of residuals, Shapiro-Wilk. Partial $\eta^{2}=0.12$ for ED and 0.16 for nervous, and negligible $(<0.034)$ for the other cases. 
Tables

Table 1

Post-experience Questionnaire on Body Representation

\begin{tabular}{|l|l|l|}
\hline No. & Variable & Statement \\
\hline Q1 & mybody & I felt as if the body I saw in the virtual world might be my body. \\
\hline Q2 & nervous & I became nervous when the other avatars approached me. \\
\hline Q3 & control & I felt like I controlled the avatar as if it was my own body. \\
\hline Q4 & notme & I felt like the avatar was not me. \\
\hline Q5 & liked & I liked being able to control the movements of the avatar. \\
\hline Q6 & greet & I wanted to say hello to the avatars as they walked past. \\
\hline
\end{tabular}

